\title{
Gene Expression and Alternative Splicing Dynamics Are Perturbed in Head Transcriptomes of Heterospecifically Mated Females
}

Fernando Diaz ( $\sim$ ferdiazfer@gmail.com )

University of Arizona

Carson Allan

University of Arizona

Therese Markow

Cinvestav UGA-Langebio

Jeremy Bono

University of Colorado Colorado Springs

Luciano Matzkin

University of Arizona

\section{Research Article}

Keywords: Speciation, postmating response, alternative splicing, intron retention, RNA-seq, head transcriptomes, D. mojavensis, D. arizonae

Posted Date: January 29th, 2021

DOl: https://doi.org/10.21203/rs.3.rs-149189/v1

License: (c) (1) This work is licensed under a Creative Commons Attribution 4.0 International License. Read Full License 


\section{Abstract \\ Background}

Despite the growing interest in the female side of copulatory interactions, the roles played by alternative splicing mechanisms of pre-RNA and the epistatic effects of these interactions on tissues outside of the reproductive tract have remained largely unknown. Here we addressed these questions in the context of con- vs heterospecific matings between Drosophila mojavensis and its sister species, $D$. arizonae. We analyzed transcriptional responses in female heads using an integrated investigation of genome-wide patterns of gene expression, including differential expression $(D E)$, alternative splicing $(A S)$ and intron retention $(I R)$.

\section{Results}

Our results indicated that early transcriptional responses were largely congruent between con- and heterospecific matings but are substantially perturbed over time. Conspecific matings induced functional pathways related to amino acid balance previously associated with the brain's physiology and female postmating behavior. Heterospecific matings often fail to activate regulation of some of these genes and induce expression of additional genes when compared with those of conspecifically-mated females. These results are consistent for all transcriptional mechanisms with some distinctions: $D E$ genes were mostly linked to pathways of proteolysis and nutrient homeostasis, while $A S$ genes are more related to photoreception and muscle assembly pathways.

\section{Conclusions}

IR seem to be an important mechanism of $D E$ regulation during the female postmating response. While $A S$ genes evolve at slower evolutionary rates than the genome background, $D E$ genes evolve at much higher rates, similar or even higher than those of seminal fluid proteins, which unveil their potential role for reproductive barriers and the extent of sexual conflict.

\section{Background}

Sexual reproduction involves a whole set of coupled interactions affecting the performance of both sexes, such as those involved in mate-recognition, male courtship and female postcopulatory responses [1]. Females undergo a complex process of physiological changes after mating called the postmating response, induced by biochemical interactions between ejaculate components transferred during copulation and female molecules in the reproductive tract $[2,3]$. The role of seminal fluid proteins in the female postmating response has been well characterized in Drosophila species, and hundreds of malederived proteins have now been identified in other taxa [4-7]. The female side has remained more elusive and very little is known about the downstream effector genes that mediate the female postmating 
response, particularly those occurring outside of the female reproductive tract (e.g. genes related to female behavior).

Although transcriptional changes induced by con- or heterospecific matings have been explored in a number of species [8-16], most of these studies have not considered alternative splicing $(A S)$ as an additional mechanism in which genes responsible for postmating changes might be regulated [17]. Differential regulation of spliced isoforms created by different combinations of exons (or intron retention $-I R$ ) from the same genomic loci may have substantial functional consequences not reflected in gene expression [18], which can uncover additional mechanisms within the complexity of molecular reproductive interactions. Recent comparisons of Drosophila species show that AS diversification contributes to lineage-specific adaptation [19], with sex-biased splicing and $I R$ rates [20] in several tissues including the brain [18], suggesting that this mechanism might be important in female behavioral responses.

Changes induced by heterospecific matings can compromise gametic interactions during the fertilization process - postmating prezygotic or PMPZ isolation [2, 21]. Moreover, conspecific matings are often accompanied by a whole set of behavioral changes such as those involved in female receptivity, exploration, diet and oviposition [22-27]. If altered by mating with a heterospecific male, these behavioral changes can compromise the mating outcome, leading to reproductive barriers. The transcriptional bases of these responses are more likely located in tissues of the central nervous system [28]. In fact, the current mechanism discovered in D. melanogaster suggests that a male-derived sex peptide - SP is not only one of the main triggers of the female response, but is also gradually released into the hemolymph by cleavage [29-31], suggesting important and lasting changes outside of the reproductive tissues. The SP interacts with a sex peptide receptor in the female (SPR), with major expression in both reproductive organs and the nervous system [32]. Consequently, transcriptional changes associated with behavioral and photoreception pathways have been detected in female heads after conspecific mating [25, 33].

It is well known that interacting male and female reproductive genes evolve rapidly [34], however it is unclear whether genes governed by $A S$ dynamics or expressed outside of the reproductive tissues follow this evolutionary path. We addressed these questions by exploring head transcriptomes in con-vs heterospecific matings between Drosophila mojavensis and $D$. arizonae. Perturbation of the female transcriptional response by heterospecific matings was first demonstrated in female tracts of Drosophila mojavensis when mating with $D$. arizonae males [8]. These species diverged $\sim 0.5 \mathrm{Mya}$ and are reproductively isolated at the gametic level, displaying strong $P M P Z$ isolation as fertilization success is reduced after heterospecific matings [21,35]. Although heterospecific mattings occur in both directions [36], transcriptional responses have not been previously explored in D. arizonae females. Here we implemented an integrated approach to explore the conspecific context of each species and demonstrate that the postcopulatory response involves functionally different roles played by $D E, A S$ and $I R$ dynamics. These responses are substantially perturbed by heterospecific matings and some of their regulatory networks evolve at male reproductive gene levels. 


\section{Results}

\section{Patterns of DE and AS}

Experimental design as described in Fig. 1, consisted of and heterospecific matings between the species $D$. mojavensis and $D$. arizonae. We obtained an average of $22 * 10^{6}$ mapped reads for each library following trimming and filtering of sequence reads. Minimum count filtering was applied independently to all different subfeatures (e.g. exon, junction, intron) at the beginning of each analysis performed. We found evidence for gene expression changes when comparing mated with virgin samples (Fig. 2a, 2b and Fig. 3). These changes were consistently detected at different hierarchies of gene expression such as at gene-wide (differential expression - $D E$ ) (Fig. 3) as well as at exon and junction features (alternative splicing - $A S$ ), including up to $16 \%$ of the $A S$ genes exhibiting differential $I R$. Although the $D E-A S$ overlap never exceeded $5 \%$ of genes, as expected from their distinct molecular regulatory mechanisms, both generally followed similar patterns in postmating experiments (Fig. 2a). However, the relative contribution of $D E$ and $A S$ showed large variation across the different conditions of the experiment. The overlap of genes responding to con- vs heterospecific matings was very low for all experiments, ranging from 14-28 \% (Fig. 2a), indicating that copulation between either $\triangle D$ moj or $\triangle D a r i$ with a heterospecific male induces a very different transcriptome response in female heads.

The female's species ( $\triangle D$ moj or $\triangle D a r i)$ seems to define the main patterns for expression responses when crossing $D$. mojavensis and $D$. arizonae. It defines the strength of the con- vs heterospecific response (Fig. 3) as well as when such changes are induced in the heads (45min vs $6 \mathrm{~h}$ postmating periods). $\triangle D$ moj crosses exhibited a larger response at $45 \mathrm{~min}$ (Fig. 3a) and higher number of genes (Fig. 2a) when compared to $\triangle D a r i$, which then tended to decrease over time. $\triangle D$ ari matings generated a response to the heterospecific matings that was slower and tended to increase over time but decreases for female heads of conspecific matings (Figs. 2a and 3b).

The direction of expression changes in mated females compared to female virgin samples showed an overrepresentation of up- vs downregulated genes (Fig. 2b). Thus, $\triangle D$ moj matings exhibited up to four times more upregulated than downregulated genes (Fig. 2b), while $₫$ Dari matings had over twice as many downregulated genes as upregulated. The number of genes and the distribution of the expression response in $D E$ genes were substantially perturbed by the heterospecific matings (Fig. 3). The response of $\triangle D$ moj was stronger for conspecific matings in terms of the number of genes involved (Fig. 2a, Fig. 3) when compared to that of heterospecific matings, while $\mathbb{D}$ Dari involved more genes in the heterospecific matings than that of the conspecific matings (Fig. 2a, Fig. 3).

\section{Transcriptional correlation between crosses and postmating periods}

We next examined the level of transcriptional correlation between con- vs heterospecific matings for genes showing significant $D E$ and $A S$ responses. Overall, $D E$ and $A S$ correlations show similar tendencies, with a strong initial correlation between con-vs heterospecific matings at $45 \mathrm{~min}$ that tended to decrease 
substantially at $6 \mathrm{~h}$ (Fig. 4). One exception was the case of $\triangle D m o j$ (con vs hetero), where $D E$ genes (Fig. 4a) showed very low correlation even at 45 min postmating (Spearman's $r=-0.16$ ), indicating substantial transcriptional perturbation in heterospecifically-mated females (Fig. 4a). $\forall$ Dari (con vs hetero) on the other hand showed a stronger transcriptional correlation at $45 \mathrm{~min}$ (Spearman's $r=0.86$ ) which then decreased at $6 \mathrm{~h}$ (Spearman's $\mathrm{r}=0.70$ ). This pattern was opposed to that observed in ASgenes (Fig. 4b), where $\triangle D$ moj showed a strong con vs hetero correlation at $45 \mathrm{~min}$, then disrupted at $6 \mathrm{~h}$, while transcriptional perturbation appeared earlier (at 45 min postmating) in $\triangle D$ Dari crosses (Fig. 4b).

We next investigated the level of correlation for genes responding to mating dynamics between the species ( $\triangle D$ moj vs $\triangle D a r i)$. With the only exception of $D E$ genes at $45 \mathrm{~min}$ (Fig. 4a), showing a moderate correlation in the conspecific-mating response between the species ( $\square D$ moj con vs $\varangle$ Dari con, Spearman's $r=0.51$ ), the rest of the comparisons $(D E$ and $A S$ ) were not correlated between the species (con- or heterospecific) (Figs. 4a and 4b).

Most of $A S$ dynamics detected through junctionSeq reflect differential isoform regulation. However, the specific case of intron retention, as detected using IRFinder, more likely indicates gene regulation by the nonsense-mediated decay $(N M D)$ or a similar pathway. We tested this hypothesis as a possible mechanism of transcriptional response to mating by estimating intron retention changes between mated vs virgin samples - IR change, for up and down-regulated genes (Fig. 5). We discovered that IR change significantly increased for down-regulated genes, while it decreased for up-regulated genes in response to all mating experiments consistently (Fig. 5). This finding is consistent with $I R$ serving as a mechanism of gene expression downregulation.

\section{Evidence of positive selection}

We investigated rates of molecular evolution $(\mathrm{w}=d N / d S)$ of $D E$ and $A S$ genes for each experimental cross. Results on evolutionary rates revealed two main patterns of molecular evolution in these genes (Fig. 6a). Firstly, $A S$ genes seem to evolve at a much lower evolutionary rate than $D E$ genes, even lower than the genome background. Second, $D E$ genes exhibited substantial differences in the evolutionary rates between both the species and crosses. The average ratio $\mathrm{w}$ is substantially higher than the genome background for conspecific matings in $D$. arizonae ( $w=0.38$, Fig. $6 a)$, while $D$. mojavensis appear to evolve at background rates. The heterospecific matings show exactly the opposite pattern, with $D E$ genes from heterospecifically-mated $D$. mojavensis females evolving more rapidly than background $(w=0.30)$, while heterospecifically-mated $D$. arizonae $D E$ genes evolving at genome background rates $(w=0.18$, Fig. 6a). Rapidly evolving $D E$ genes appear to evolve at similar rates or even higher than those of seminal fluid proteins previously reported by Kelleher et al. (2009) in D. mojavensis (Fig. 6).

\section{Functional analysis}

To analyze the functional pathways associated with female genes responding to mating experiments in female heads, we performed gene ontology $(G O)$ enrichment analysis. We found that detected genes are enriched by four main functional pathways (Fig. $6 \mathrm{~b}$ and $6 \mathrm{c}$ ): i) nutrient homeostasis, ii) chitin metabolism, iii) photoreception and iv) muscle assembly (Fig. 6). From these, pathways associated with 
i) nutrient homeostasis are particularly interesting for their implications in the female postmating response. These pathways are all associated with amino acid balance in brains: L-Carnitine from Lysine and Methionine (Carnitine biosynthetic process and Gamma-butyrobetaine dioxygenase activity, Fig. 6b), and the production of Threonine and Leucine (Threonine and Leucine biosynthetic process, Fig. 6b). These pathways are all associated with nutrient homeostasis in brains and some of their metabolic functions have been previously detected in conspecific mating experiments in D. melanogaster [28, 38]. Physiological functions associated with these specific amino acids include energy balance in brain tissues (i.e. Carnitine) $[39,40]$, insulin secretion following food intake, increasing cellular uptake of nutrients (i.e. Leucine) [41] and female behaviors such as sleep suppression (i.e. Threonine) [42, 43].

We found functional specialization between $D E$ and $A S$ genes, with $D E$ patterns (Fig. 6b) being more associated with i) and ii) types of pathways, while $A S$ genes (Fig. 6c) were dominated by iii) and iv). Both mechanisms of gene regulation showed dramatic functional differentiation between con- vs heterospecific crosses (Fig. 6b and 6c). Most of enriched pathways were detected in a conspecific context, but only a subset remained significant in the heterospecific matings (Figs. $6 \mathrm{~b}$ and $6 \mathrm{c}$ ). Genes associated with i) female behavioral and iii) photoreception, activated in conspecific matings are not activated in heterospecifically-mated $\triangle D$ Dmoj. Similarly, all proteolytic pathways activated in conspecific ¿Dari were not activated in heterospecific matings. Genes that have been previously reported as related with the i) female behavior were exclusive to $D$. mojavensis and were not enriched in $D$. arizonae (Fig. $6 \mathrm{~b}$ ).

\section{Discussion}

The cactophilic $D$. mojavensis and $D$. arizonae are promiscuous, mating multiple times a day, even heterospecifically in lab settings (Diaz et al unpublished data). Yet these species have remained isolated for $\sim 0.5 \mathrm{My}$, suggesting the presence of multiple reproductive barriers preventing introgressive hybridization $[35,66,67]$. $P M P Z$ isolation has been confirmed for crosses involving $D$. mojavensis females [21], where the reaction mass is more evident and molecular interactions in the female lower reproductive tract were found altered by the heterospecific ejaculate [8]. Here, we demonstrate that copulation induces substantial transcriptional changes in head tissues of females that are substantially perturbed when mating with a heterospecific male in both species. These changes compromise functional pathways important for the female postcopulatory physiology and behavior that normally would be expressed in conspecifically-mated females, some of these evolving rapidly, which might have implications for the extent of sexual conflict [68].

Our results indicate that mating induces not only gene expression changes in female heads soon after mating, but also that a great part of this response is controlled by alternative splicing. The number of genes responding through $A S$ often exceeded that of $D E$ genes, but both mechanisms appear to be involved in the female postcopulatory response. We examined $A S$ patterns caused by multiple mechanisms, including intron retention - IR [18], when comparing mated vs virgin females. Differential usage of examined gene features showed substantial consequences for the postmating response that were not reflected in gene expression of head transcriptomes. The role of $A S$ in the postmating response 
has not been previously evaluated, but is consistent with the complexity of epistatic interactions related to sexual traits [34]. Interestingly, in this study, genes experiencing $D E$ or $A S$ appear to be almost mutually exclusive (less than $5 \%$ overlap). Consequently, the female postmating response seems to target different functions through each of these mechanisms. $D E$ genes are mainly linked to pathways of proteolysis and nutrient homeostasis, while $A S$ genes are more related to those involved in photoreception and muscle assembly changes.

$I R$ is a particular case of $A S$ that, although has been associated with some active functional changes, is most likely linked to gene regulation resulting in degradation of the mRNA by the nonsense-mediated mRNA decay - NMD pathway $[20,69]$. We demonstrate that $I R$ is not only differentially regulated between con- and heterospecific matings but seems to be an active mechanism of gene regulation as $I R$ rates increased for down regulated genes but decreased for up-regulated genes.

The transcriptional response to mating has been studied in a few insect species [9-16], showing biologically meaningful pathways common to the postmating response across different species. In fact, some of the mating-activated genes that we found in female heads are associated with functional pathways previously reported in different tissues and species. Proteolytic pathways for example, are within the most common and strongly activated genes that are part of the female response, found in both reproductive tissues and whole female bodies $[10,38,70]$. However, this is a complex reproductive response, given that a great part of the male ejaculate is also composed of a diverse cocktail of both proteases and their inhibitors [2].

Increasing interest in SFP have revealed a great array of proteases with diverse functions in both testis and the female postmating response $[4,12,71]$. Most of these are related to earlier postmating processes and molecular interactions occurring in the female tract (e.g. sperm storage and SFP cleavage) [71-73], but it is unclear whether the proteases or inhibitors expressed by the female are involved in the same functions. However, the lasting effects, even several days after mating, and the fact that they have been detected in several species, from insects to mammals [4,73], suggest that these proteolytic cascades are involved in multiple functions of the whole organism mating response. For example, the expression of proteolytic cascades we observed in heads of mated females does not seem connected to sperm-related functions that occur in the female reproductive tract. One possibility is that some of these cascades are involved in protein degradation for amino acid related pathways and nutrient homeostasis, a hypothesis supported by our functional analysis. We identified two functional categories associated with Carnitine biosynthesis. This particular amino acid is known to start with the degradation of proteins containing $\mathrm{N}$ methylated lysine by proteolytic cascades [74], with a major role in energy homeostasis in the nervous system $[39,40]$.

We detected several biosynthetic pathways associated with the production of specific amino acids important for nutrient homeostasis such as carnitine, threonine, and leucine. Although such pathways are not directly classified as behavioral or reproductive, some of their metabolic functions have been previously detected in conspecific experiments in $D$. melanogaster $[28,38]$. These networks regulate 
energy balance [39, 40] and nutritional uptake in brain tissues [41], and have been directly linked to several postcopulatory behaviors, including circadian rhythms [42, 43], nutrient sensing, exploratory behavior for specific nutrient source, consumption and posterior oviposition $[26,27,75,76]$. Consequently, mated $D$. melanogaster females experience a major switch in their diet following copulation [77], consuming more amino acids during the dark phase [26]. The mechanisms of this behavioral switch have been linked to neuronal signaling triggered by SP-SPR dynamics in D. melanogaster [78-80].

Transcriptional dynamics detected in female heads of both species were substantially perturbed by heterospecific matings. Thus, changes of regulatory networks related to nutrient homeostasis in brain tissues were characteristic of conspecific dynamics in $D$. mojavensis, while changes detected in $D$. arizonae females were dominated by proteolytic pathways. The biological function of genes, and the magnitude of their expression were perturbed following copulation with a heterospecific male. Heterospecifically-mated $D$. mojavensis were unable to activate nutrient-homeostasis- ( $D E$ genes) and photoreception-related ( $A S$ genes) pathways and activated proteolytic and some muscle assembly genes instead. In contrast, heterospecifically-mated $D$. arizonae females showed no enriched pathways for $D E$ genes, suggesting strong functional perturbation. These results, suggest that interactions between seminal fluid components and the female tract induce distant epistatic effects in tissues of the female head soon after mating $[28,38]$. The mode of signaling for these changes in insects is still an ongoing investigation. Given the distance and heterogeneity of the tissues involved, changes induced in the female head are assumed to be the product of altered interaction networks, influencing the physiology of other tissues $[29,81]$. This is more an indirect effect of neurons associated with internal reproductive tissues, which may produce signaling molecules that influence gene expression at distant sites in the fly body [82]. However, evidence from $D$. melanogaster suggests that seminal fluid components like the $S P$ rapidly circulate in the female's hemolymph and has been found associated with brain tissues [32]. Furthermore, immediate post-copulatory transcriptional responses in the nervous system could also be due to social interactions, as has been shown in $D$. melanogaster with males that court but fail to copulate [83]. Further research is needed to disentangle the underlying mechanisms causing the activation of genes outside of the female reproductive tract and their involvement in the post-copulatory response.

The male side of mating has been long been investigated in several species and it is well known that male reproduction genes, and particularly seminal fluid proteins - SFP evolve rapidly [34]. Although the female genes have only been investigated in a few species, results in $D$. mojavensis and $D$. virilis female's reproductive tracts $[66,70,84]$ indicated that these genes not always evolve as rapid as the male genes. Here, we found that $D E$ genes in female heads are evolving rapidly in $D$. arizonae, but not in $D$. mojavensis. Conspecific genes detected in $D$. arizonae are related to different proteolytic pathways and showed evolutionary rates even higher than SFP genes previously detected in these species [37]. These pathways were not enriched in $D$. mojavensis females, but some increased when mated with $D$. arizonae males. $A S$ genes on the other hand exhibited evolutionary rates even lower than the genome background. These genes were linked to very specific functions of highly conserved genes. Therefore, this could be due to the conservation of those specific gene functions in these species, but more likely seems to be 
reflecting a general trend in the molecular evolution of $A S$ genes [85]. To date, the role of $A S$ in the female postmating response has not previously evaluated in insect species, nor the relationship between $A S$ and molecular evolution in Drosophila. However, this same result was also previously found when investigating strictly AS genes across the mouse and human genomes [85], suggesting that the level of alternative splicing and molecular evolution at the sequence level are negatively correlated. Constitutive exons of alternative isoforms tend to evolve faster than newly alternatively spliced exons $[85,86]$. It is unclear how these heterogenic patterns would affect gene-wide molecular evolution or specific gene families in insects, but highly constrained exons could decrease molecular evolution at the gene level. These genes are also presumed more pleiotropic as alternative isoforms can evolve without major changes in sequence through functional specialization of different exon arrays.

\section{Conclusions}

We demonstrate that the female postmating response is a complex process that involves the general female physiology as well as strong tissue-specific transcriptional changes with variation in strength and direction [38]. By implementing an integrated transcriptional analysis in head tissues of mated females between $D$. mojavensis and $D$. arizonae, we were able to reveal previously unknown roles played by $D E, A S$ and $I R$, and how these dynamics are integrated through functional specializations within the female postmating response. Substantial transcriptional perturbations by heterospecific matings suggests a previously unknown role of these genes in postmating barriers that rely on female behavioral changes triggered during copulation. The consequences of sexual conflict have always been looked in the face of genes that are more directly linked to reproduction (i.e. SFP and female tract genes) [68]. We provide evidence showing that genes expressing in distant head tissues and likely involved in female postcopulatory behavior, evolve at male reproductive gene levels (or higher). Given the functions of genes involved, these changes might be costly to the female, affecting not only fertilization, but also might compromise the hybrid performance if fertilization succeed but nutritional and egg-laying decisions are compromised. These types of changes have been detected in a few species [87-89], but their transcriptional bases and evolutionary rates have remained unknown. Our results indicate that transcriptional perturbation following heterospecific mating extends beyond the female reproductive tract. The extent of these interactions might be larger than previously thought, opening the door to investigate the link between head transcriptomes and reproductive barriers in con- vs heterospecifically mated females.

\section{Methods}

\section{Samples and matting experiments}

All experiments were carried out using $D$. mojavensis and $D$. arizonae isofemale lines originally collected from Anza Borrego Desert State Park, Borrego Springs, CA (in 2002) and Guaymas, Sonora, Mexico (in 2000), respectively. Inbred lines were held at $25^{\circ} \mathrm{C}$, under $12: 12 \mathrm{~h}$ light:dark cycle and controlled density conditions in 8-dram glass vials with banana-molasses media [44] for all stocks and experiments. 


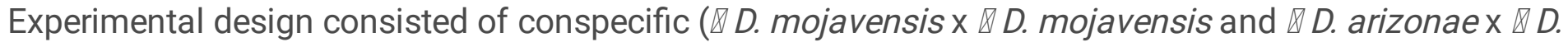

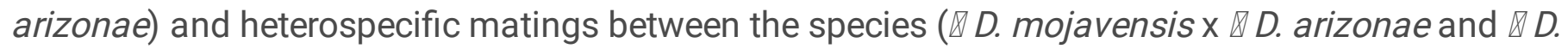
arizonaex $\triangle D$. mojavensis) (Fig. 1). We refer to the reciprocal mating as $\triangle D m o j$ for matings involving $D$. mojavensis female and $₫ D$ ari for those involving $D$. arizonae female. All mating experiments were performed using between 7-10 days old virgin flies by isolating single pairs in vials constantly inspected for copulation events during a 2-hour window immediately after the incubator lights turned on. Males were removed from vials after copulation and females were kept in the vial until the specified postmating period was reached ( $45 \mathrm{~min}$ or $6 \mathrm{hrs}$ ), when heads were collected from both mated and control virgin females (Fig. 1). Groups of 20 specimens were pooled for each sample and three replicates were considered per experimental cross, which generated 15 samples for each direction of the cross ( $\triangle D$ Dmoj and $\triangle D$ Dari -con/-het) for a total of 30 samples. All tissues were placed immediately in TRIzol and kept at $-80^{\circ} \mathrm{C}$ until total RNA extractions.

\section{RNA extraction, cDNA library construction and sequencing}

Total RNA was extracted using Direct-zol RNA kit (Zymo Research). Both RNA quality and quantity were inspected on a Bioanalyzer (Applied Biosystems/Ambion). cDNA libraries were created using KAPA Stranded mRNA-Seq Kit according to the manufacturer's instructions. Libraries were sequenced at Novogene Inc. using the HiSeq SBS v4 High Output Kit on Illumina platform flow cells with runs of $2 x$ 150 bp paired-end reads. Illumina's HiSeq Control Software and CASAVA software (Illumina, Inc.) were used for base calling and sample demultiplexing.

\section{Sequence trimming and mapping}

Nearly 700 million total paired-end read sequences were obtained from the Illumina runs. Reads were trimmed for quality and adapter sequences were removed using a minimum quality base of $Q=20$ and minimum read length of $50 \mathrm{bp}$ using the software Trimmomatic [45]. Trimmed reads were then mapped to corresponding reference genomes using splice-aware mapper GSNAP [46] with the option of new splice events detection. The $D$. mojavensis reference genome was used for samples involving $\triangle D$ moj, while $D$. arizonae was used as reference for $\triangle D a r i$ samples. Generated sam files were converted to bam format after indexing and filtering for a minimum mapping quality of $M Q=20$ using SAMtools [47]. These mapping results were then used for all differential expression and alternative splicing downstream pipelines.

\section{Reference genomes}

Template based genomes were used for mapping RNAseq reads. For $D$. mojavensis, the assembly from [48] (Accession number SRP190536) was used with updated annotations retrieved from FlyBase version FB2016_05 [49]. A template genome version of $D$. arizonae (osf.io/ukexv) was assembled using the same method as D. mojavensis in [48] with paired-end and mate pair Illumina reads from [50] (Accession number SRP278895). 


\section{Differential expression - DE}

We created a gene level read count matrix for all samples using featureCounts [51]. The read count matrix was filtered for a minimum count cutoff $=3 \mathrm{cpm}$ over at least two out of three replicates per comparable group. All $D E$ analyses were performed using the R package edgeR [52] after $T M M$ library normalization. Normalized counts were analyzed by Generalized Linear Models (GLM) accounting for negative binomial variable of read counts, followed by $D E$ analyses. All comparisons were performed between mated females (con- and heterospecific mating) and virgin females ( $\square$ Dmoj and $\triangle D$ Dari) at each postmating period ( $45 \mathrm{~min}$ and $6 \mathrm{~h}$ ). An $F D R=0.05$ correction [53] for multiple comparisons as well as a log-foldchange threshold of 1.0 was applied to all $P$-values.

\section{Alternative splicing - AS}

We used the JunctionSeq [54] pipeline in order to detect genome-wide patterns of alternative spliced genes. $A S$ is defined as the relative regulation of isoforms belonging to a multi-isoform gene with respect to a given biological condition [55]. The pipeline is based on differential usage calculated from both exon and junction feature coverages. The pipeline relies on the originally implemented method in DEXSeq [56], which tested differential usage of annotated exons, but extended to splice junctions usage and both annotated and non-annotated splicing events. A new flattened GTF annotation file where overlapping features are not allowed was first generated using QoRTs [55]. All overlapping genes were merged as composed by a flat set of non-overlapping exons and splice junctions with unique identifiers. QoRTs was also used to generate a read count matrix for $A S$ analysis, including three types of read counts per gene as estimated by exons, junction and gene level counts. Generated count matrix was then used by JunctionSeq R package [54] to estimate differential exon and junction usage with respect to gene-wide expression. No read was counted more than once in the model since exon and junction dispersions are then fitted independently. As for differential expression analysis, alternatively spliced genes were then detected if at least one exon or splice junction was differentially used between mated females (con- and heterospecific mating) and virgin females ( $\triangle D$ moj and $\triangle D a r i)$ at each postmating period (45 min and $6 \mathrm{~h}$ ). An $F D R=0.01$ correction [53] for multiple comparisons was applied to all $P$-values.

\section{Intron retention rates - IR}

Intron retention is a specific type of $A S$ that is not necessarily captured by JunctionSeq and can have different biological implications that help to better explain expression changes. An intron can be retained in the final mature $m R N A$, coding for a new function $[57,58]$ or a nonfunctional transcript that is degraded by nonsense-mediated decay (NMD) [59]. We investigated whether postmating $A S$ events also involve mechanisms of intron retention using the IRFinder pipeline [60]. A new reference annotation was built by removing all overlapping features present in the same strain sense of individual introns and then unique identifiers were assigned to each flattened exon. Only regions with high mapping scores as estimated through simulated reads across the genome are identified and included in the flattened annotation file. A read count matrix with all reads overlapping splice junctions was generated and $I R$ rates were estimated as: junction reads / (junction reads + intronic reads) for each sample. The count matrix 
was then used by IRFinder R package [60] in order to estimate GLM. This method is used to test the fold change of $I R$ between biological conditions using the DESeq2 R package framework [61]. Genes with differential $I R$ were then detected if least one intron was differentially retained between mated females (con- and heterospecific mating) and virgin females ( $\triangle D$ moj and $\triangle D$ Dari) at each postmating period (45 min and 6h). An $F D R=0.05$ correction [53] for multiple comparisons was applied to all $P$-values.

\section{Statistical analysis of genes under $D E, A S$ and IR}

A Spearman's correlation matrix comparing relative expression levels of significant $D E$ genes was generated in order to investigate the relationship of gene expression changes between con-vs heterospecific matings as well as between postmating periods ( $45 \mathrm{~min}$ vs $6 \mathrm{~h}$ ) and the two directions of the cross ( $\triangle D$ moj vs $\triangle D a r i)$. Because $I R$ changes are more likely linked to mechanisms of downregulation by transcript degradation, we tested this hypothesis by estimating $I R$ changes between mated vs virgin samples - IR change, while comparing up vs down-regulated genes. A GLM analysis was performed using categories of up and down regulation as independent variables and the level of $I R$ change dependent the dependent variable for each mating experiment. GLM analysis was performed after square root transformation to account for normal distribution and homoscedasticity.

\section{Functional and evolutionary analyses}

Overrepresentation of specific categories of biological functions were then investigated for $D E$ and $A S$ genes using GOseq R package framework [62]. Additionally, we investigated signatures of positive selection on genes responding to con-vs heterospecific matings as well as for each of the overrepresented categories detected. For this, we estimated evolutionary rates ( $\left.w=d_{n} / d_{s}\right)$ using codeml, part of PAML 4.9 [63]. CDS alignments between $D$. mojavensis and $D$. arizonae were produced with MUSCLE 3.8.31 [64]. Any alignments with internal stop codons or frameshifts were removed before analysis. Codem/ was run using model $O$ with default values. Raw synonymous and nonsynonymous polymorphism counts were generated with KaKs Calculator 1.2 [65]. We further extracted putative seminal fluid genes identified in a proteomic analysis of $D$. mojavensis male accessory glands [37] and compare their rate of molecular evolution with those that were differentially regulated in heads. A GLM analysis was performed to compare the average evolutionary rates under each mating experiment against the genome background rates or each mating experiment. GLM analysis was performed after square root transformation to account normal distribution and homoscedasticity.

\section{List Of Abbreviations}

$D E$

Differential expression

AS

Alternative splicing

IR 
Intron retention

¿Dmoj

female $D$. mojavensis

QDari

female $D$. arizonae

QDnav

female $D$. navojoa

\section{Declarations}

\section{Ethics approval and consent to participate}

This section is not applicable to the present study.

\section{Consent for publication}

This section is not applicable to the present study.

\section{Availability of data and materials}

A template genome version of $D$. arizonae and $D$. mojavensis was deposited in public repository (link for editors and reviewers: https://osf.io/ukexv/?view_only=7e20375d605040e4add37f707cbabaf4v), while all RNAs-seq reads have been deposited in the Sequence Read Archive under accession number (link for editors and reviewers: (https://dataview.ncbi.nlm.nih.gov/object/PRJNA693826?

reviewer=krjn5pt4ejuuehnlhbhhkeiccs). Both links will be publicly available upon acceptance.

\section{Competing interests}

The authors declare that they have no competing interests.

\section{Funding}

The research presented in this publication was supported by the Eunice Kennedy Shriver National Institute of Child Health and Human Development of the National Institutes of Health under award number R21HD097545 to LMM and JMB as well as by funds from the University of Arizona to LMM.

\section{Authors' contributions}

JMB, LMM and TAM conceived the initial idea. The project was designed by FD, JMB and LMM. FD performed most of the fly work, library construction and transcriptomic analyses. CWA participated in fly and molecular work and performed molecular evolution analyses. All authors participated in the data analysis and the writing of the manuscript. All authors read and approved the final manuscript.

\section{Acknowledgements}


We would like to thank Joshua M. Coleman and undergraduate students Nathaniel Talamantes, Rorie Shae Robinson, Kamal Jitendra Patel, Moruj Athma and Graham Wegner from the University of Arizona for assistance in the Drosophila part of this project.

\section{Literature Cited}

1. Dimijian GG. Evolution of Sexuality: Biology and Behavior. Baylor Univ Med Cent Proc. 2005;18:24458.

2. Wolfner MF. Battle and ballet: Molecular interactions between the sexes in drosophila. J Hered. 2009;100:399-410.

3. Manier MK, Lüpold S, Belote JM, Starmer WT, Berben KS, Ala-Honkola O, et al. Postcopulatory sexual selection generates speciation phenotypes in drosophila. Curr Biol. 2013;23:1853-62.

4. Avila FW, Sirot LK, LaFlamme BA, Rubinstein CD, Wolfner MF. Insect Seminal Fluid Proteins: Identification and Function. Annu Rev Entomol. 2011;56:21-40. doi:10.1038/jid.2014.371.

5. Ahmed-Braimah YH, Unckless RL, Clark AG. Evolutionary dynamics of male reproductive genes in the drosophila virilis subgroup. G3 Genes, Genomes, Genet. 2017;7:3145-55.

6. Mueller JL, Ravi Ram K, McGraw LA, Bloch Qazi MC, Siggia ED, Clark AG, et al. Cross-species comparison of Drosophila male accessory gland protein genes. Genetics. 2005;171:131-43.

7. Dottorini T, Nicolaides L, Ranson H, Rogers DW, Crisanti A, Catteruccia F. A genome-wide analysis in Anopheles gambiae mosquitoes reveals 46 male accessory gland genes, possible modulators of female behavior. Proc Natl Acad Sci U S A. 2007;104:16215-20.

8. Bono JM, Matzkin LM, Kelleher ES, Markow TA. Postmating transcriptional changes in reproductive tracts of con- and heterospecifically mated Drosophila mojavensis females. Proc Natl Acad Sci U S A. 2011;108:7878-83.

9. Liu PC, Hao DJ. Behavioural and transcriptional changes in post-mating females of an egg parasitoid wasp species. R Soc Open Sci. 2019;6.

10. Mack PD, Kapelnikov A, Heifetz Y, Bender M. Mating-responsive genes in reproductive tissues of female Drosophila melanogaster. Proc Natl Acad Sci U S A. 2006;103:10358-63.

11. Thailayil J, Gabrieli P, Caputo B, Bascuñán P, South A, Diabate A, et al. Analysis of natural female post-mating responses of Anopheles gambiae and Anopheles coluzzii unravels similarities and differences in their reproductive ecology. Sci Rep. 2018;8:1-10.

12. Alfonso-Parra C, Ahmed-Braimah YH, Degner EC, Avila FW, Villarreal SM, Pleiss JA, et al. MatingInduced Transcriptome Changes in the Reproductive Tract of Female Aedes aegypti. PLoS Negl Trop Dis. 2016;10:1-24.

13. Kocher SD, Richard FJ, Tarpy DR, Grozinger CM. Genomic analysis of post-mating changes in the honey bee queen (Apis mellifera). BMC Genomics. 2008;9:232.

14. Gao B, Song XQ, Yu H, Fu DY, Xu J, Ye H. Mating-Induced Differential Expression in Genes Related to Reproduction and Immunity in Spodoptera litura (Lepidoptera: Noctuidae) Female Moths. J Insect 
Sci. 2020;20.

15. Fowler EK, Bradley T, Moxon S, Chapman T. Divergence in Transcriptional and Regulatory Responses to Mating in Male and Female Fruitflies. Sci Rep. 2019;9:1-15. doi:10.1038/s41598-019-51141-9.

16. Al-Wathiqui N, Dopman EB, Lewis SM. Postmating transcriptional changes in the female reproductive tract of the European corn borer moth. Insect Mol Biol. 2016;25:629-45.

17. Telonis-Scott M, Kopp A, Wayne ML, Nuzhdin S V., Mclntyre LM. Sex-specific splicing in Drosophila: Widespread occurrence, tissue specificity and evolutionary conservation. Genetics. 2009;181:42134.

18. Venables JP, Tazi J, Juge F. Regulated functional alternative splicing in Drosophila. Nucleic Acids Res. 2012;40:1-10.

19. Gibilisco L, Zhou Q, Mahajan S, Bachtrog D. Alternative Splicing within and between Drosophila Species, Sexes, Tissues, and Developmental Stages. PLoS Genet. 2016;12:1-19.

20. Wang M, Branco AT, Lemos B. The $Y$ chromosome modulates splicing and sex-biased intron retention rates in Drosophila. Genetics. 2018;208:1057-67.

21. Kelleher ES, Markow TA. Reproductive tract interactions contribute to isolation in Drosophila. Fly (Austin). 2007;1:33-7.

22. Bloch Qazi MC, Wolfner MF. An early role for the Drosophila melanogaster male seminal protein Acp36DE in female sperm storage. J Exp Biol. 2003;206:3521-8.

23. Neubaum DM, Wolfner MF. Mated Drosophila melanogaster females require a seminal fluid protein, Acp36DE, to store sperm efficiently. Genetics. 1999;153:845-57.

24. Sirot LK, Wolfner MF, Wigby S. Protein-specific manipulation of ejaculate composition in response to female mating status in Drosophila melanogaster. Proc Natl Acad Sci U S A. 2011;108:9922-6.

25. Hollis B, Koppik M, Wensing KU, Ruhmann H, Genzoni E, Erkosar B, et al. Sexual conflict drives male manipulation of female postmating responses in Drosophila melanogaster. Proc Natl Acad Sci U S A. 2019;116:8437-44.

26. Uchizono S, Tabuki Y, Kawaguchi N, Tanimura T, Itoh TQ. Mated Drosophila melanogaster females consume more amino acids during the dark phase. PLoS One. 2017;12:1-16.

27. Corrales-Carvajal VM, Faisal AA, Ribeiro C. Internal states drive nutrient homeostasis by modulating exploration-exploitation trade-off. Elife. 2016;5:1-29.

28. Dalton JE, Kacheria TS, Knott SRV, Lebo MS, Nishitani A, Sanders LE, et al. Dynamic, mating-induced gene expression changes in female head and brain tissues of Drosophila melanogaster. BMC Genomics. 2010;11.

29. Swanson WJ. Sex peptide and the sperm effect in Drosophila melanogaster. Proc Natl Acad Sci U S A. 2003;100:9643-4.

30. Peng J, Chen S, Bu S, Liu H, Honegger T, Kubli E. Gradual Release of Sperm Bound Sex-Peptide Controls Female Postmating Behavior in Drosophila. Curr Biol. 2005;15:207-13. 
31. Ram KR, Wolfner MF. Sustained post-mating response in Drosophila melanogaster requires multiple seminal fluid proteins. PLoS Genet. 2007;3:2428-38.

32. Yapici N, Kim YJ, Ribeiro C, Dickson BJ. A receptor that mediates the post-mating switch in Drosophila reproductive behaviour. Nature. 2008;451:33-7.

33. Delbare SYN, Chow CY, Wolfner MF, Clark AG, Wilson Sayres M. Roles of Female and Male Genotype in Post-Mating Responses in Drosophila melanogaster. J Hered. 2017;108:740-53.

34. Swanson WJ, Vacquier VD. The rapid evolution of reproductive proteins. Genetics. 2002;3:137-44.

35. Knowles LL, Markow TA. Sexually antagonistic coevolution of a postmating-prezygotic reproductive character in desert Drosophila. Proc Natl Acad Sci U S A. 2001;98:8692-6.

36. Lopez-Maestre H, Carnelossi EAG, Lacroix V, Burlet N, Mugat B, Chambeyron S, et al. Identification of misexpressed genetic elements in hybrids between Drosophila-related species. Sci Rep. 2017;7 December 2016:1-13. doi:10.1038/srep40618.

37. Kelleher ES, Watts TD, LaFlamme BA, Haynes PA, Markow TA. Proteomic analysis of Drosophila mojavensis male accessory glands suggests novel classes of seminal fluid proteins. Insect Biochem Mol Biol. 2009;39:366-71. doi:10.1016/j.ibmb.2009.03.003.

38. Newell NR, Ray S, Dalton JE, Fortier JC, Kao JY, Chang PL, et al. The drosophila post-mating response: Gene expression and behavioral changes reveal perdurance and variation in cross-tissue interactions. G3 Genes, Genomes, Genet. 2020;10:967-83.

39. Laranjeira A, Schulz J, Dotti CG. Genes related to fatty acid $\beta$-oxidation play a role in the functional decline of the drosophila brain with age. PLoS One. 2016;11:1-18.

40. Schulz JG, Laranjeira A, Van Huffel L, Gärtner A, Vilain S, Bastianen J, et al. Glial $\beta$-Oxidation regulates drosophila energy metabolism. Sci Rep. 2015;5:1-9.

41. Ziegler AB, Manière G, Grosjean Y. Jhl-21 plays a role in Drosophila insulin-like peptide release from larval IPCs via leucine transport. Sci Rep. 2018;8:1-11.

42. Sonn JY, Lee J, Sung MK, Ri H, Choi JK, Lim C, et al. Serine metabolism in the brain regulates starvation-induced sleep suppression in Drosophila melanogaster. Proc Natl Acad Sci U S A. 2018;115:7129-34.

43. Ki Y, Lim C. Sleep-promoting effects of threonine link amino acid metabolism in Drosophila neuron to GABAergic control of sleep drive. Elife. 2019;8:1-24.

44. Coleman JM, Benowitz KM, Jost AG, Matzkin LM. Behavioral evolution accompanying host shifts in cactophilic Drosophila larvae. Ecol Evol. 2018;8:6921-31.

45. Bolger AM, Lohse M, Usadel B. Trimmomatic: A flexible trimmer for Illumina sequence data. Bioinformatics. 2014;30:2114-20.

46. Wu TD, Nacu S. Fast and SNP-tolerant detection of complex variants and splicing in short reads. Bioinformatics. 2010;26:873-81.

47. Li H, Handsaker B, Wysoker A, Fennell T, Ruan J, Homer N, et al. The Sequence Alignment/Map format and SAMtools. Bioinformatics. 2009;25:2078-9. 
48. Allan CW, Matzkin LM. Genomic analysis of the four ecologically distinct cactus host populations of Drosophila mojavensis. BMC Genomics. 2019;20:1-13.

49. Gramates LS, Marygold SJ, Dos Santos G, Urbano JM, Antonazzo G, Matthews BB, et al. FlyBase at 25: Looking to the future. Nucleic Acids Res. 2017;45:D663-71.

50. Sanchez-Flores A, Peñaloza F, Carpinteyro-Ponce J, Nazario-Yepiz N, Abreu-Goodger C, Machado CA, et al. Genome evolution in three species of cactophilic drosophila. G3 Genes, Genomes, Genet. 2016;6:3097-105.

51. Liao Y, Smyth GK, Shi W. featureCounts: An efficient general purpose program for assigning sequence reads to genomic features. Bioinformatics. 2014;30:923-30.

52. Robinson MD, McCarthy DJ, Smyth GK. edgeR: A Bioconductor package for differential expression analysis of digital gene expression data. Bioinformatics. 2009;26:139-40.

53. Benjamini Y, Hochberg Y. Controlling the false discovery rate: a practical and powerful approach to multiple testing. J R Stat Soc. 1995;57:298-300.

54. Hartley SW, Mullikin JC. Detection and visualization of differential splicing in RNA-Seq data with JunctionSeq. Nucleic Acids Res. 2016;44:e127.

55. Hartley SW, Mullikin JC. QoRTs: A comprehensive toolset for quality control and data processing of RNA-Seq experiments. BMC Bioinformatics. 2015;16:224. http://dx.doi.org/10.1186/s12859-0150670-5.

56. Anders S, Reyes A, Huber W. Detecting differential usage of exons from RNA-seq data. Genome Res. 2012;22:2008-17.

57. Jacob AG, Smith CWJ. Intron retention as a component of regulated gene expression programs. Hum Genet. 2017;136:1043-57.

58. Monteuuis G, Wong JJL, Bailey CG, Schmitz U, Rasko JEJ. The changing paradigm of intron retention: Regulation, ramifications and recipes. Nucleic Acids Res. 2019;47:11497-513.

59. Farlow A, Meduri E, Dolezal M, Hua L, Schlötterer C. Nonsense-mediated decay enables intron gain in Drosophila. PLoS Genet. 2010;6:1-7.

60. Middleton R, Gao D, Thomas A, Singh B, Au A, Wong JJL, et al. IRFinder: Assessing the impact of intron retention on mammalian gene expression. Genome Biol. 2017;18:51.

61. Love MI, Huber W, Anders S. Moderated estimation of fold change and dispersion for RNA-seq data with DESeq2. Genome Biol. 2014;15:550.

62. Young MD, Wakefield MJ, Smyth GK, Oshlack A. Gene ontology analysis for RNA-seq: accounting for selection bias. Genome Biol. 2010;11:R14.

63. Yang Z. PAML 4: Phylogenetic analysis by maximum likelihood. Mol Biol Evol. 2007;24:1586-91.

64. Edgar RC. MUSCLE: Multiple sequence alignment with high accuracy and high throughput. Nucleic Acids Res. 2004;32:1792-7.

65. Zhang Z, Li J, Zhao X-Q, Wang J, Gane K-SW, Yu J. KaKs_Calculator: Calculating Ka and Ks through model selection and model averaging. Genomics Proteomics Bioinforma. 2006;4:259-63. 
66. Kelleher ES, Swanson WJ, Markow TA. Gene duplication and adaptive evolution of digestive proteases in Drosophila arizonae female reproductive tracts. PLoS Genet. 2007;3:1541-9.

67. Machado CA, Matzkin LM, Reed LK, Markow TA. Multilocus nuclear sequences reveal intra- and interspecific relationships among chromosomally polymorphic species of cactophilic Drosophila. Mol Ecol. 2007;16:3009-24.

68. Dapper AL, Wade MJ. Relaxed Selection and the Rapid Evolution of Reproductive Genes. Trends Genet. 2020;36:640-9. doi:10.1016/j.tig.2020.06.014.

69. Graveley BR, Brooks AN, Carlson JW, Duff MO, Landolin JM, Yang L, et al. The developmental transcriptome of Drosophila melanogaster. Nature. 2011;471:473-9.

70. Ahmed-braimah YH, Wolfner MF, Clark AG. Differences in post-mating transcriptional responses between conspecific and heterospecific matings in Drosophila. bioRxiv. 2020;:1-28.

71. Karr TL. Fruit flies and the sperm proteome. Hum Mol Genet. 2007;16:124-33.

72. Sitnik J, Gligorov D, Maeda R, Karch F, Wolfner MF. The female post-mating response requires genes expressed in the secondary cells of the male accessory gland in Drosophila melanogaster. Genetics. 2016;202:1029-41.

73. LaFlamme BA, Ravi Ram K, Wolfner MF. The Drosophila melanogaster seminal fluid protease “Seminase” regulates proteolytic and post-mating reproductive processes. PLoS Genet. 2012;8:30-2.

74. Strijbis K, Vaz FM, Distel B. Enzymology of the carnitine biosynthesis pathway. IUBMB Life. 2010;62:357-62.

75. Wang $G$ hang, Wang $L$ ming. Recent advances in the neural regulation of feeding behavior in adult Drosophila. J Zhejiang Univ Sci B. 2019;20:541-9.

76. Owusu-Ansah E, Perrimon N. Modeling metabolic homeostasis and nutrient sensing in Drosophila: Implications for aging and metabolic diseases. DMM Dis Model Mech. 2014;7:343-50.

77. Kubli E. Sexual behavior: Dietary food switch induced by sex. Curr Biol. 2010;20:R474-6. doi:10.1016/j.cub.2010.04.038.

78. Ribeiro C, Dickson BJ. Sex peptide receptor and neuronal TOR/S6K signaling modulate nutrient balancing in Drosophila. Curr Biol. 2010;20:1000-5. doi:10.1016/j.cub.2010.03.061.

79. Bowman E, Tatar M. Reproduction regulates Drosophila nutrient intake through independent effects of egg production and sex peptide: Implications for aging. Nutr Heal Aging. 2016;4:55-61.

80. Gioti A, Wigby S, Wertheim B, Schuster E, Martinez P, Pennington CJ, et al. Sex peptide of Drosophila melanogaster males is a global regulator of reproductive processes in females. Proc $\mathrm{R}$ Soc B Biol Sci. 2012;279:4423-32.

81. Yang C, Rumpf S, Xiang Y, Gordon MD, Song W, Jan Y, et al. Control of the Postmating Behavioral Switch in Drosophila Females by Internal Sensory Neurons. Neuron. 2009;61:519-26.

82. Häsemeyer M, Yapici N, Heberlein U, Dickson BJ. Sensory Neurons in the Drosophila Genital Tract Regulate Female Reproductive Behavior. Neuron. 2009;61:511-8. 
83. Carney GE. A rapid genome-wide response to Drosophila melanogaster social interactions. BMC Genomics. 2007;8:2-11.

84. Bono JM, Matzkin LM, Hoang K, Brandsmeier L. Molecular evolution of candidate genes involved in post-mating-prezygotic reproductive isolation. J Evol Biol. 2015;28:403-14.

85. Cusack BP, Wolfe KH. Changes in alternative splicing of human and mouse genes are accompanied by faster evolution of constitutive exons. Mol Biol Evol. 2005;22:2198-208.

86. Modrek B, Lee CJ. Alternative splicing in the human, mouse and rat genomes is associated with an increased frequency of exon creation and/or loss. Nat Genet. 2003;34:177-80.

87. Matute DR. The magnitude of behavioral isolation is affected by characteristics of the mating community. Ecol Evol. 2014;4:2945-56.

88. McLain DK, Pratt AE. The cost of sexual coercion and heterospecific sexual harassment on the fecundity of a host-specific, seed-eating insect (Neacoryphus bicrucis). Behav Ecol Sociobiol. 1999;46:164-70.

89. Quinõnes-Lebrón SG, Kralj-Fišer S, Gregoric M, Lokovšek T, Candek K, Haddad CR, et al. Potential costs of heterospecific sexual interactions in golden orbweb spiders (Nephila spp.). Sci Rep. 2016;6:4-9.

\section{Figures}




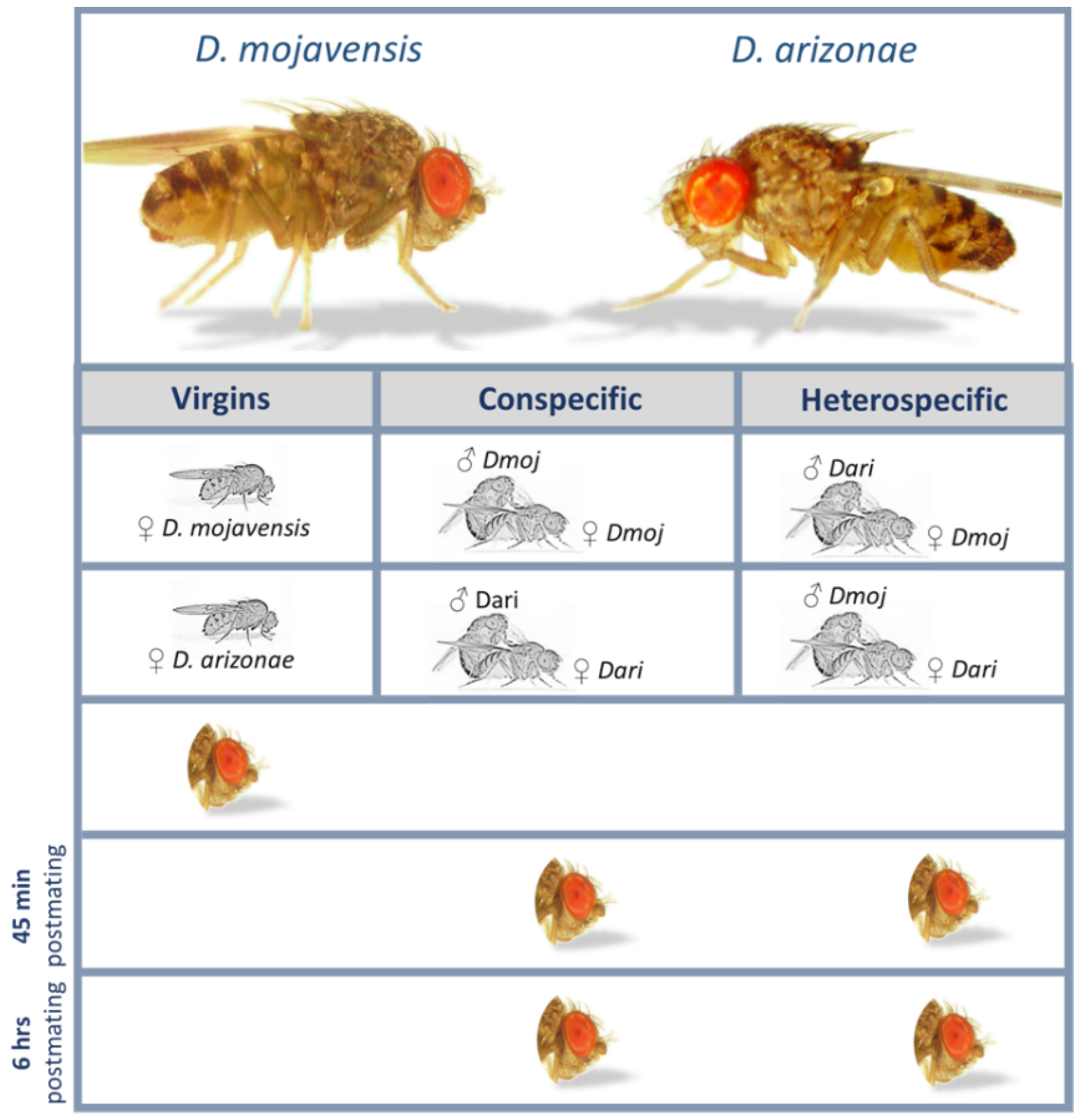

Figure 1

Experimental design for con- and heterospecific matings between D. mojavensis and D. arizonae. RNAseq libraries were constructed for head tissues of virgins, con- and heterospecifically mated females (45min and $6 \mathrm{hrs}$ postmating). 


\section{a) AS and DE}

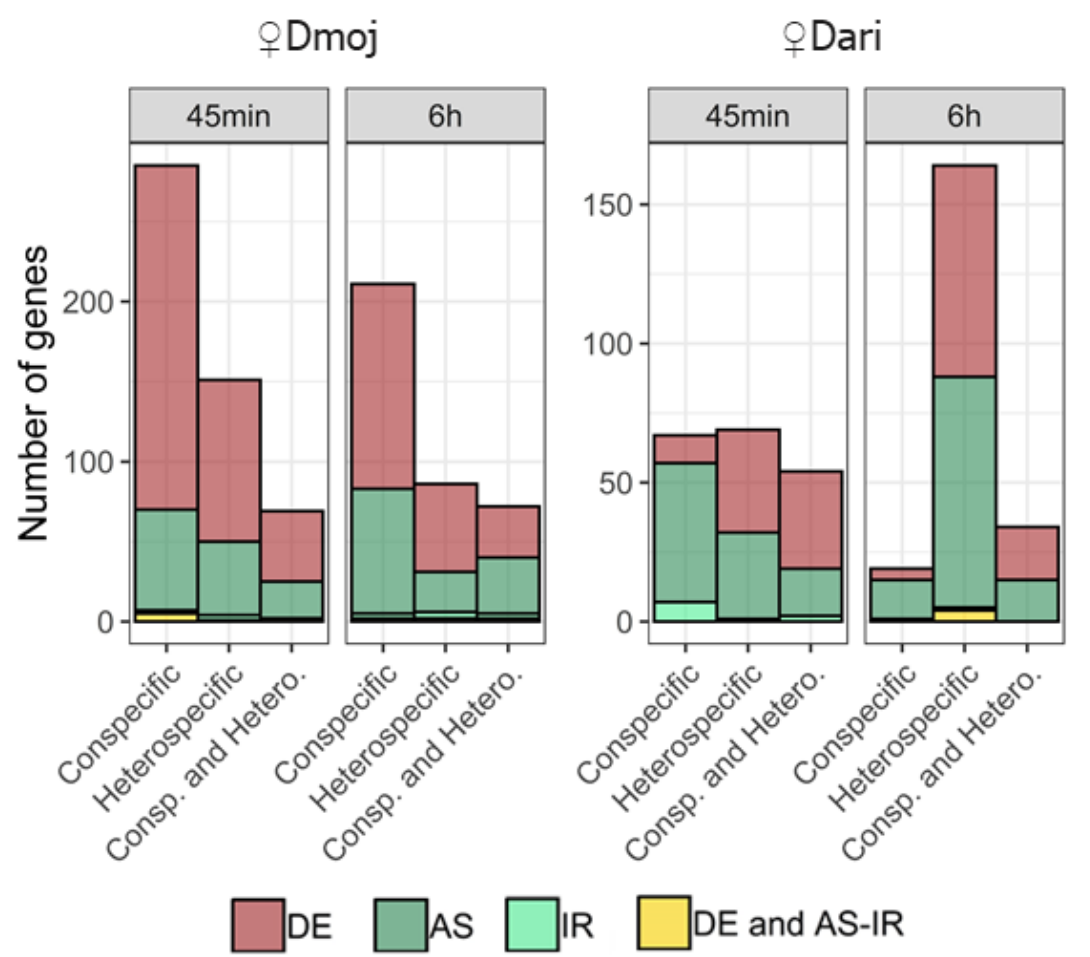

\section{b) Direction of $\mathrm{DE}$}
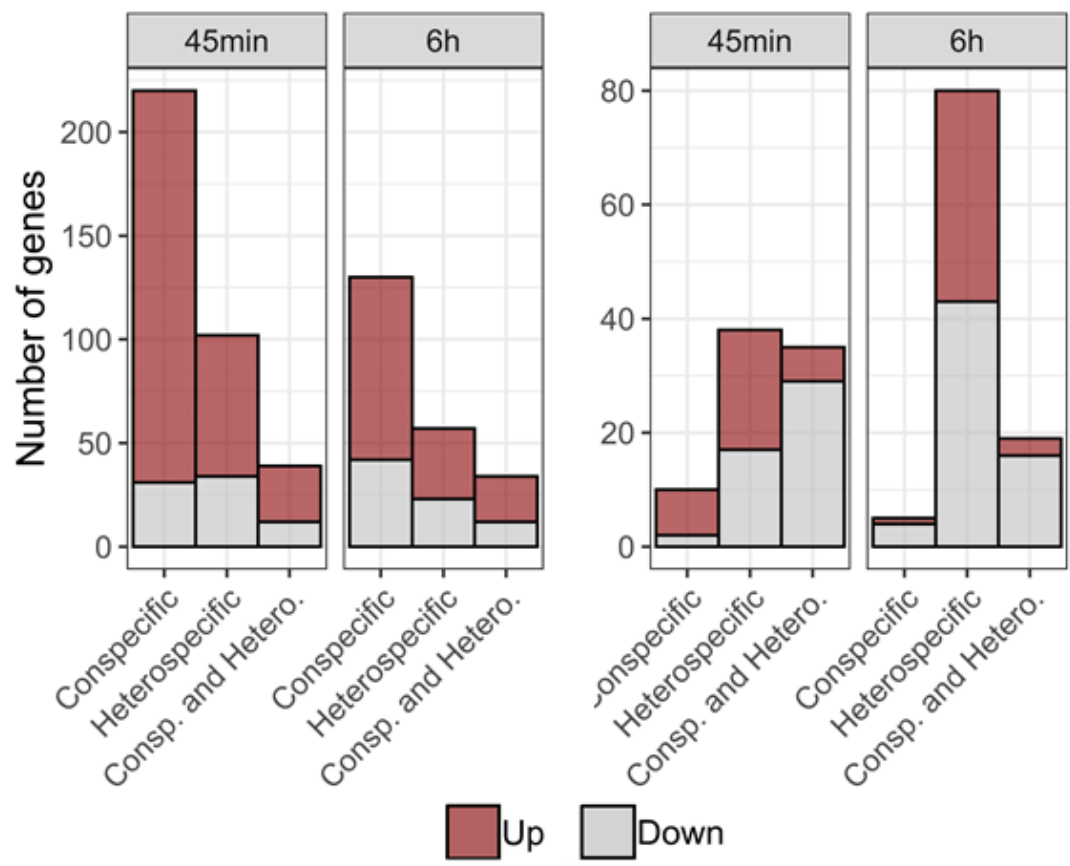

\section{Figure 2}

Patterns of gene expression in head transcriptomes of females following con- and heterospecific matings between D. mojavensis and D. arizonae. All comparisons are performed against virgin females, showing the number of genes with significant DE and AS (including IR) after FDR $<0.05$ and threshold corrections for con- and heterospecific crosses involving a) D. mojavensis females ( $\triangle \mathrm{Dmoj}$ ) and b) D. arizonae 
females ( $\triangle \mathrm{Dmoj})$ after $45 \mathrm{~min}$ and $6 \mathrm{~h}$ postmating. Since the overlap is indicated, the color points represent significant DE exclusive of each comparison (e.g. Conspecific only).

a) Dmoj
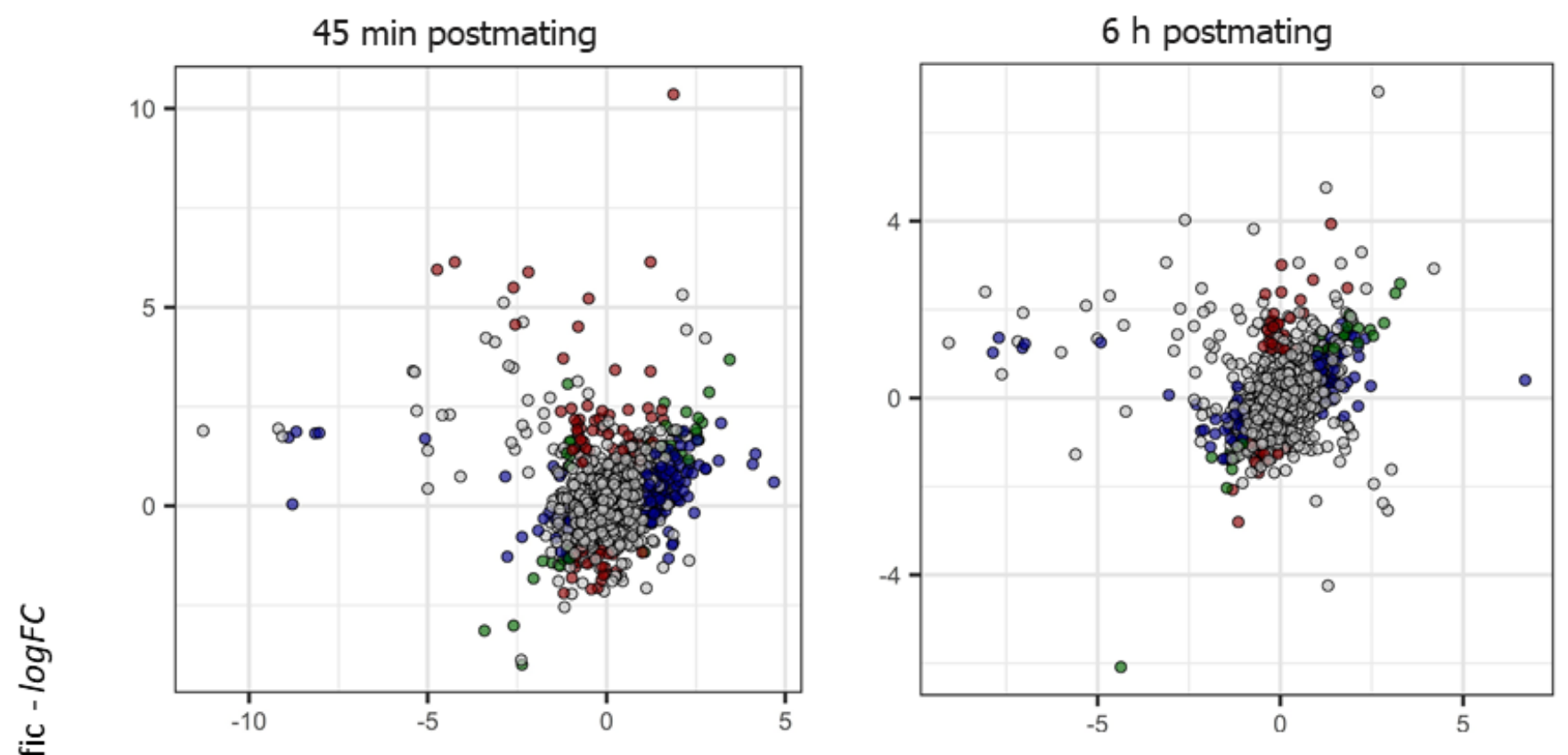

b) ○Dari
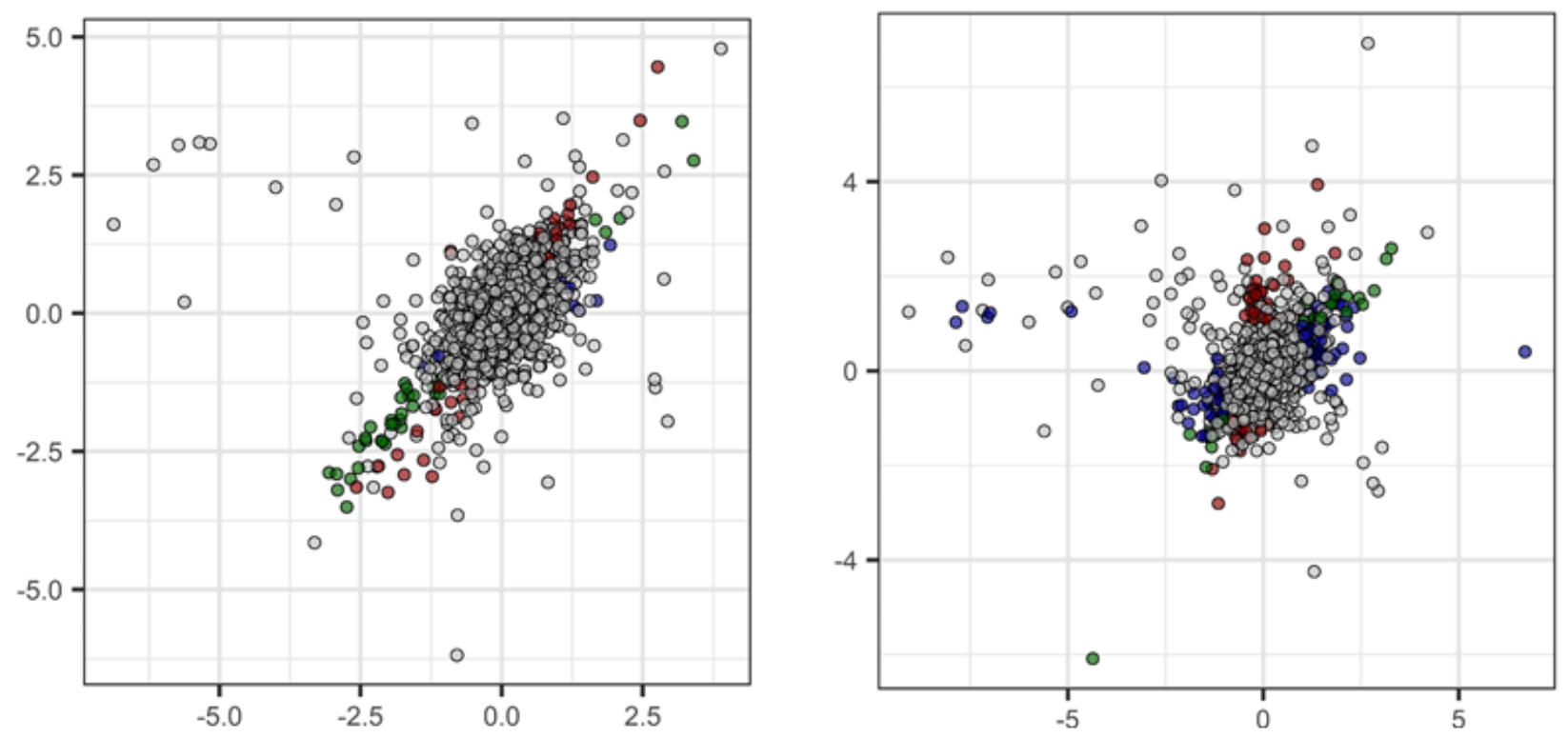

Conspecific - $\log F C$

- 1. Overlap ○ 2. Conspecific ○ 3. Heterospecific ○ 4. No-DE

\section{Figure 3}

Differential expression for head transcriptomes of females following con- and heterospecific matings between D. mojavensis and D. arizonae. All DE comparisons are performed against virgin females, showing the number and expression change of significant DE genes after FDR $<0.05$ and threshold 
corrections for a) $\triangle \mathrm{Dmoj}$ and b) $\triangle \mathrm{D}$ ari. Scatterplots represent the relation of relative gene expression (log2FC) between con- vs heterospecific matings. The relationship is represented for $45 \mathrm{~min}$ and $6 \mathrm{~h}$ postmating. Genes with significant DE and their overlaps are indicated. The number of significant DE genes for each of these categories is represented in the barplots on the right, indicating the direction of $D E$ change with respect to that in virgin samples. Since the overlap is indicated, the color points represent significant DE exclusive of each comparison (e.g. Conspecific only).

\section{a) Differential expression}

b) Alternative splicing
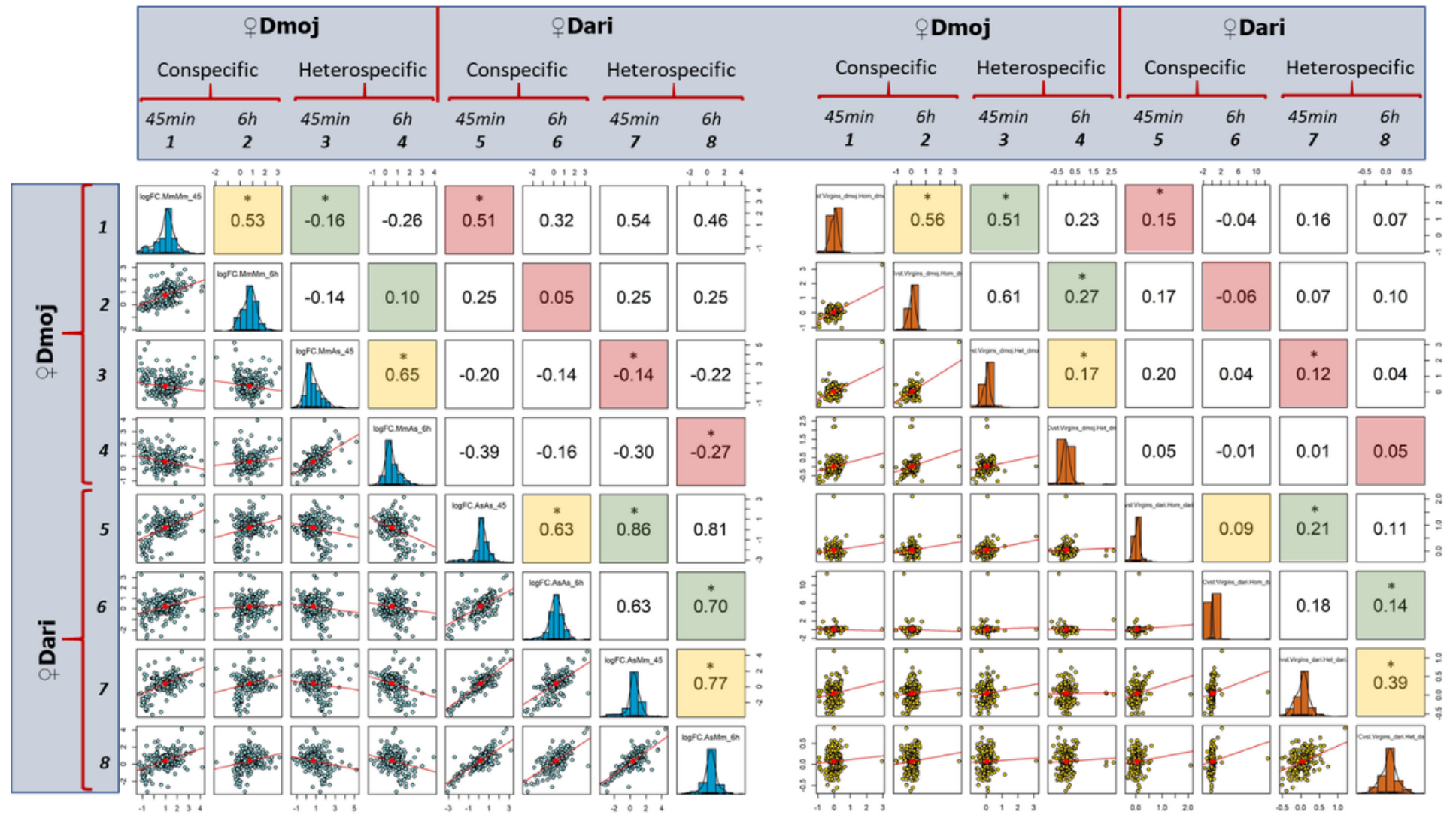

\section{Figure 4}

Relationship of transcriptional responses between con- vs heterospecifically mated females of D. mojavensis and D. arizonae. Pairwise correlation coefficient matrix (Spearman's r) was estimated only for genes with significant a) Differential expression - DE (FDR < 0.05) and b) AS genes (FDR < 0.01), comparing log2 $\mathrm{FC}$ across all mating experiments. Biologically meaningful correlations are highlighted in yellow for those correlations between $45 \mathrm{~min}$ vs $6 \mathrm{~h}$ postmating periods, green for comparisons between con- vs heterospecific matings and pink for those correlations between the two directions of the cross

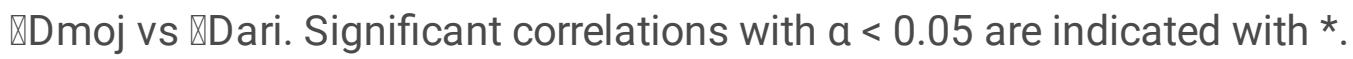



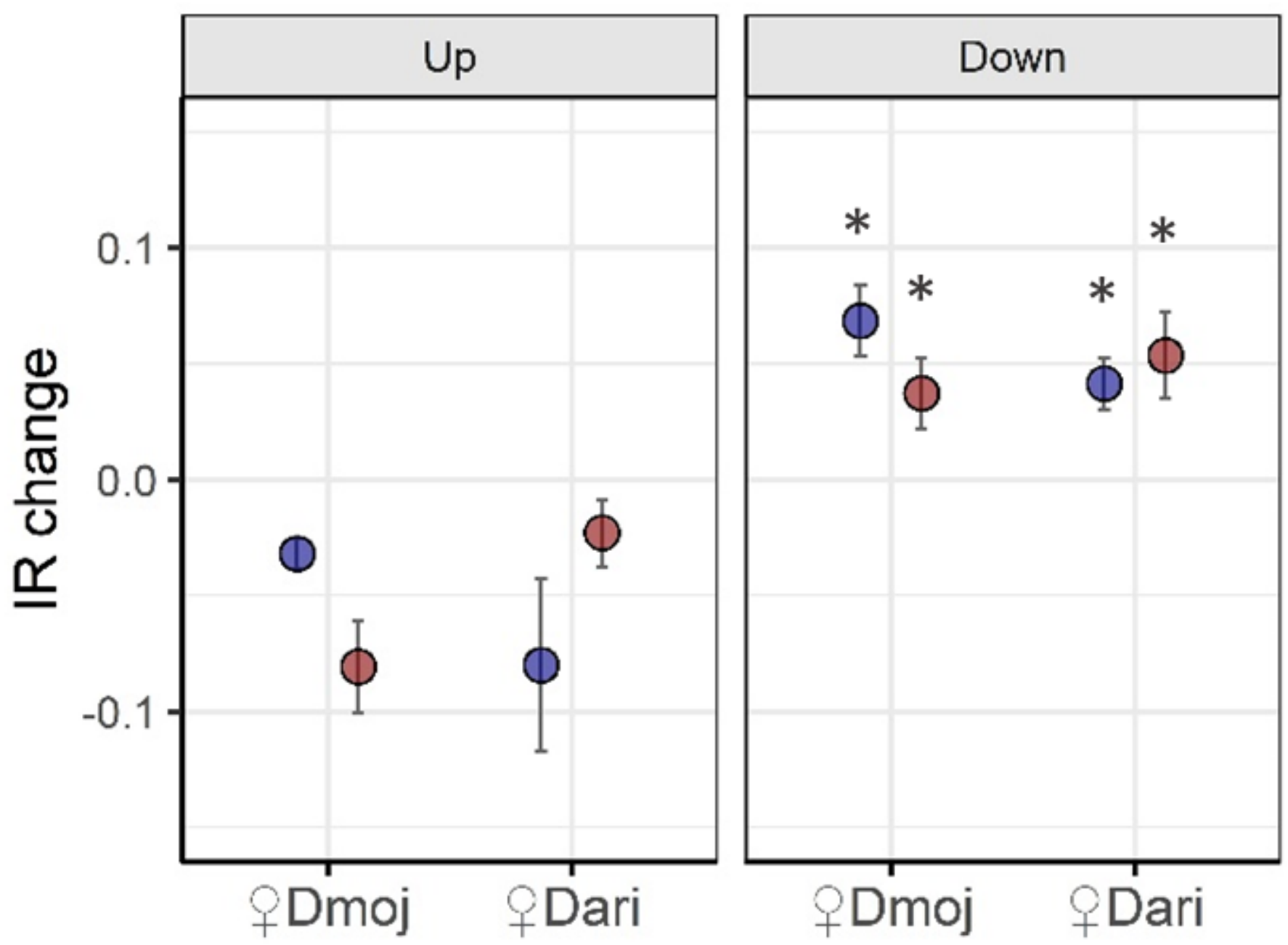

\section{- Conspecific \\ - Heterospecific}

Figure 5

Intron retention - IR change as estimated for up- and down-regulated genes detected for con- and heterospecific matings between D. mojavensis and D. arizonae. IR change was estimated as IR mated IR virgin samples. All mating experiments showed significant increase in of IR rates for down-regulated genes with respect to that of up-regulated ones. All significant comparisons with $a<0.05$ following GLM analysis are indicated with * in the "down" plot. 
a) Evolutionary rates

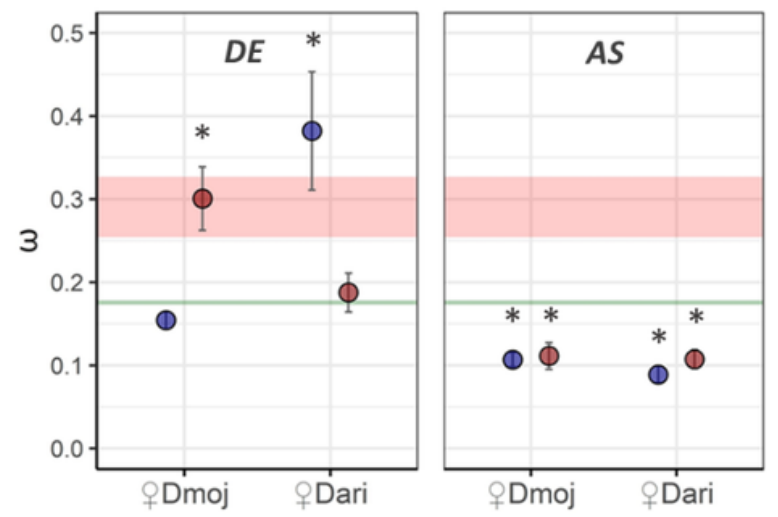

c) Alternatively spliced genes

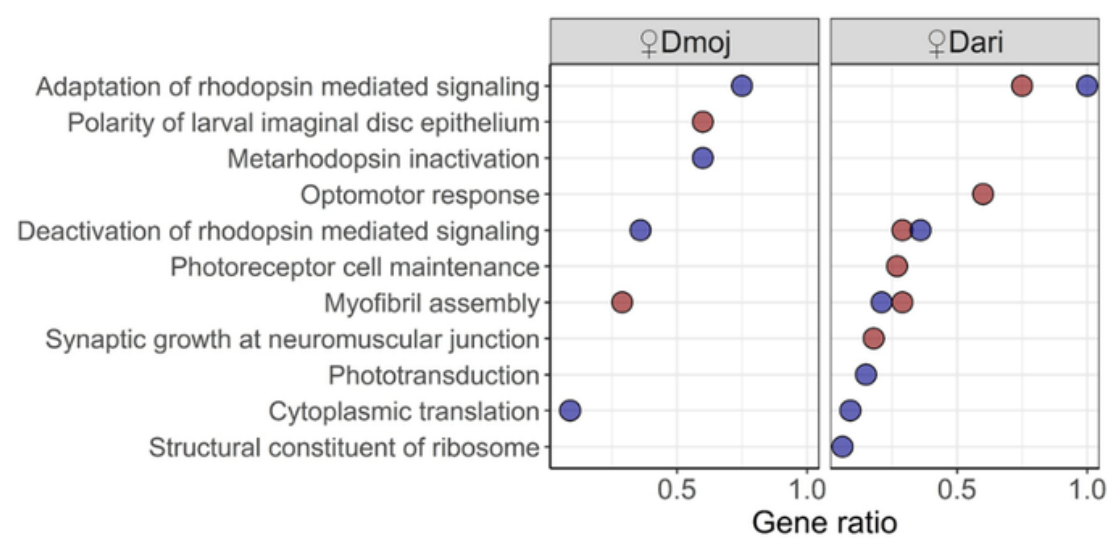

b) Differentially expressed genes
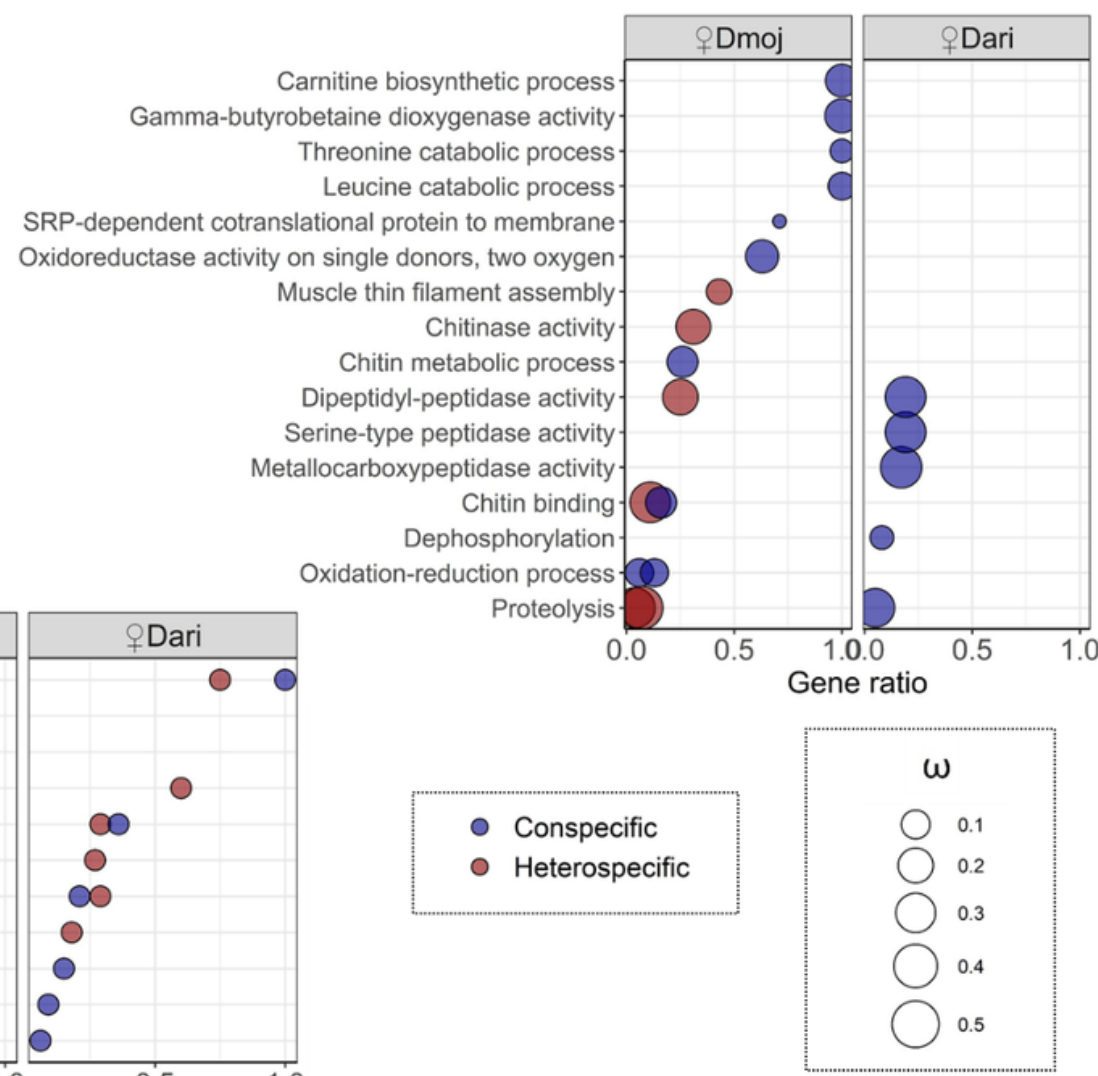

\section{Figure 6}

Evolutionary dynamics and functional analysis of female DE and AS genes induced by mating. a) Average pairwise $w$ between D. mojavensis and D. arizonae head genes. The variation of $w$ for the genome background (green) and SFPs (pink) are is indicated. These genes are a subset of accessory gland-biased genes that contain a predicted signal sequence following Kelleher et al. (2009). Error bars represent standard error of the mean. Significant comparisons from genome background rates using following $a<0.05$ in a GLM analysis are indicated with *. Functional analysis is shown for b) DE and c) AS genes, indicating gene ontology enrichment categories for con- and heterospecific matings between the species. The gene ratio of detected genes within each enriched category is indicated. All significant comparisons with following GLM analysis are indicated with * in the "down" plot. 